\title{
Digitales Lernen und Lehren: Führt Corona zu einer zeitgemäßen Bildung?
}

\author{
Linda Grogorick (D) - Susanne Robra-Bissantz
}

Eingegangen: 27. September 2021 / Angenommen: 14. Oktober 2021 / Online publiziert: 2. November 2021

(C) Der/die Autor(en) 2021

Zusammenfassung Zum Schutz vor Corona musste das Lernen und Lehren an Hochschulen, Schulen sowie anderen Aus- und Weiterbildungseinrichtungen zeitweise nahezu vollständig ins Digitale überführt werden. Doch auch schon vor Corona hat kaum noch jemand ausschließlich analog, also ohne digitale Unterstützung, gelernt. Das (digitale) Lernen befindet sich bereits seit längerem im Wandel. Deswegen widmet sich dieser Beitrag einerseits der Thematik, wie sich das Lernen im Allgemeinen sowie mögliche Lernformate gewandelt haben und andererseits, wie Corona das Lernen an Schulen, Hochschulen sowie anderen Aus- und Weiterbildungseinrichtungen verändert hat.

Schlüsselwörter Digitales Lernen · Lerntheorien · Lernformate · Hochschule · Schule $\cdot$ Aus- und Weiterbildung

Linda Grogorick $(\bowtie) \cdot$ Susanne Robra-Bissantz

Lehrstuhl Informationsmanagement, Institut für Wirtschaftsinformatik, Technische Universität

Braunschweig, Mühlenpfordtstr. 23, 38106 Braunschweig, Deutschland

E-Mail: 1.grogorick@tu-bs.de

Susanne Robra-Bissantz

E-Mail: s.robra-bissantz@tu-bs.de 


\title{
Digital Learning and Teaching: Does Corona lead to Modern Education?
}

\begin{abstract}
Learning and teaching at universities, schools and other training facilities had to be digitally substituted by online courses almost completely to protect against Corona. But even before Corona, almost no one learned exclusively analog anymore, i.e. without digital support. (Digital) learning has been in a state of transformation for quite some time now. For this reason, this article focuses on how learning in general and possible learning formats have changed in the last years, and on how Corona has currently transformed learning at schools, universities, and other training facilities.
\end{abstract}

Keywords Digital Learning · Learning Theories · Learning Format • University · School · Training Facilities

\section{Einleitung}

Die Wissens- und Kompetenzvermittlung an Hochschulen und Schulen sowie anderen Aus- und Weiterbildungseinrichtungen hat sich durch eine zunehmende Digitalisierung in den vergangenen Jahren stark verändert und steht derzeit vor allem durch die Unterbrechungen des regulären Lehrbetriebs zum Schutz vor Corona im gesamtgesellschaftlichen Fokus.

Digitales Lernen und Lehren musste abrupt nahezu überall zur Normalität werden. Doch auch schon vor der Umstellung der Aus- und Weiterbildung aufs Digitale hat kaum noch jemand rein analog, also ohne Internet und Laptop, gelernt. Die Vermittlung von Wissen, Fähigkeiten und Kompetenzen im Bildungswesen befindet sich demnach bereits seit längerem im Wandel, wobei Corona diesen noch zusätzlich beschleunigt hat und deswegen als Chance für ein zeitgemäßes Lernen und Lehren angesehen werden kann.

Damit eine zunehmende Digitalisierung des Lernens und Lehrens gelingt, müssen sich Lehrende und Lernende, aber auch das Umfeld, anpassen und weiterentwickeln. Eine Digitalisierung der Lerninhalte alleine ist nicht ausreichend, um eine gelungene Transformation der Lehre zu realisieren. Analoge Formen der Wissensvermittlung sind nicht direkt ins Digitale überführbar. Obwohl analog und digital zwar häufig die gleichen Inhalte erlernt werden sollen, muss dies doch auf unterschiedliche Art und Weise erfolgen, da eine digitale Aufnahme von neuem Wissen andere Wege erforderlich macht als eine analoge Wissensaufnahme. Neben der Gestaltung innovativer Lehr- und Lernkonzepte schließt dies beispielsweise auch Lernumgebungen, wie die Förderung einer Gemeinschaft mit regem Austausch und Kontakt, ein.

In dem vorliegenden Beitrag schauen wir uns vor diesem Hintergrund einmal an, wie sich das (digitale) Lernen in den vergangenen Jahren entwickelt hat. Nicht nur die Art und Weise, wie man lernt, was durch Lerntheorien beschrieben wird, hat sich verändert, sondern auch die Aufgaben der digitalen Medien innerhalb der Lernprozesse, wodurch neben klassischen Lernformaten auch neuere möglich geworden sind. Die in Folge von Corona plötzliche und vor allem fast vollständige 
Umstellung des Lernens und Lehrens ins Digitale hat ebenfalls einige Veränderungen hervorgebracht, weshalb wir uns der Thematik widmen, wie Corona das Lernen an Hochschulen, Schulen sowie weiteren Aus- und Weiterbildungseinrichtungen gewandelt hat.

\section{Digitales Lernen}

Digitales Lernen, häufig auch als E-Learning (elektronisches Lernen) bezeichnet, hat sich in den vergangenen Jahren rasant entwickelt und verändert sich auch weiterhin stetig. Vor vielen Jahren wurde der Begriff E-Learning eingeführt und von Kerres (2013, S. 6) wie folgt definiert: „E-Learning ist ein Oberbegriff für alle Varianten der Nutzung digitaler Medien zu Lehr- und Lernzwecken, sei es auf digitalen Datenträgern oder über das Internet, etwa um Wissen zu vermitteln, für den zwischenmenschlichen Austausch oder das gemeinsame Arbeiten an digitalen Artefakten“".

Im Alltag verwenden wir digitale Medien heutzutage ganz selbstverständlich, beispielsweise beim Vorlesen lassen eines Rezeptes durch einen Sprachassistenten oder bei der Routenplanung zu unserem Urlaubsziel. Digitale Medien sind längst zu einem festen Bestandteil unseres Lebens geworden. Folglich nehmen digitale Medien auch beim Lernen eine immer zentralere Rolle ein, wodurch eine entsprechende Nutzung bei der Planung und Gestaltung von Lehr- und Lernangeboten zunehmend berücksichtigt und mitgedacht wird.

Damit einhergehend ändert sich auch das allgemeine Verständnis vom Lernen. Eine künstliche Trennung zwischen einem eher traditionellen, häufig mit Präsenzunterricht gleichgestellten, und mediengestützten Lernen existiert nicht mehr, vielmehr gehen beide Formen eine Verbindung ein. Dadurch sind digitale Medien nicht länger nur ein Zusatz, sondern bilden einen integralen sowie zentralen Bestandteil bei der Gestaltung von Lernumgebungen.

Digitale Medien entwickeln sich rasant. Ständig sind neue Tools verfügbar, wobei sich nicht direkt feststellen lässt, welche davon mit einem hohen Nutzen einhergehen und sich am Markt durchsetzen, und welche in kürzester Zeit wieder vom Markt verschwunden sind. Dennoch ist häufig sogar eine kostenlose Verwendung entsprechender Tools möglich, so dass die Lehre kreativ und unter Berücksichtigung didaktischer Aspekte gestaltet werden kann. Im Kern sollte es dabei immer darum gehen, Lehren und Lernen besser zu machen.

\subsection{Lerntheorien und Aufgaben digitaler Medien}

Auf welche Art und Weise gelernt wird, also Wissen vermittelt und darüber Fähigkeiten sowie Kompetenzen aufgebaut werden, thematisieren unterschiedliche Lerntheorien. Diese haben sich in den vergangenen Jahren ebenso verändert wie die Aufgaben digitaler Medien beim Lernen. Abb. 1 gibt darüber einen einleitenden Überblick.

Geprägt wurden die Lerntheorien viele Jahre vor allem durch die Ansätze des Behaviorismus, Kognitivismus und Konstruktivismus. Doch erklären diese Ansätze 
Lerntheorien

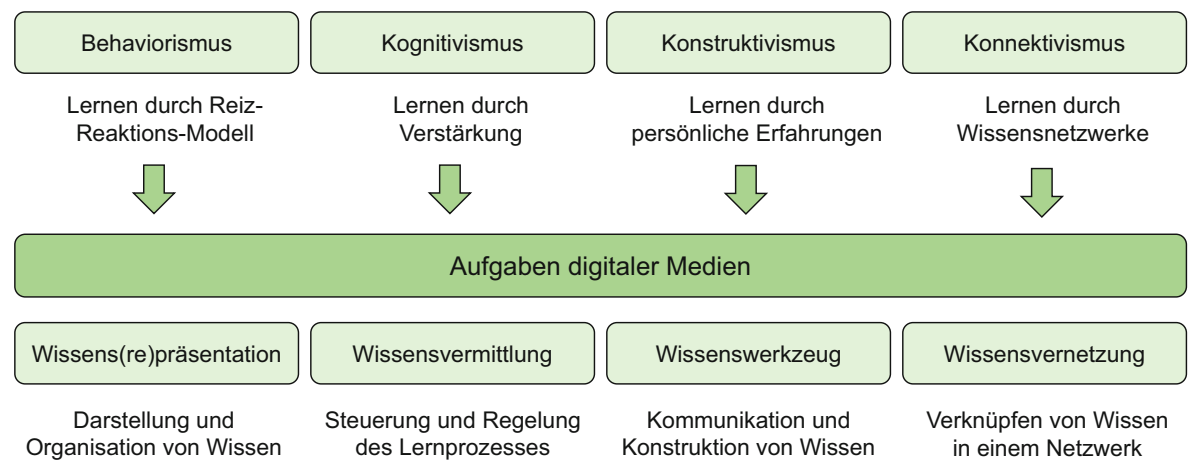

Abb. 1 Lerntheorien und Aufgaben digitaler Medien

hauptsächlich den Lernprozess selbst und vernachlässigen dabei weitestgehend Erfahrungswissen und Impulse durch andere Personen. Die heutige Informations- und Wissensgesellschaft ist aber insbesondere dadurch geprägt, dass einzelne Personen nicht mehr alles wissen und alle Erfahrungen selbstständig sammeln können, so dass das eigene Wissen in einem Prozess des lebenslangen Lernens daher stetig selbstgesteuert angepasst werden muss. Vor diesem Hintergrund bietet der Ansatz des Konnektivismus wichtige Anregungen für die Gestaltung des Lernens und Lehrens.

\subsubsection{Behaviorismus}

Die Kernannahme der behavioristischen Lerntheorie (z. B. vertreten durch Pavlov, Watson und Skinner) besteht darin, dass Lehrende wissen, was Lernende wissen sollten und folglich zu lernen haben. Bei dieser Theorie gibt es eine klassische Rollenverteilung mit einer nahezu einseitigen Sender-Empfänger-Beziehung zwischen den aktiven Lehrenden und eher passiven Lernenden. Das bedeutet im Sinne eines Reiz-Reaktions-Modells, dass Lehrende einen geeigneten Stimulus (Reiz) präsentieren, um bei den Lernenden anschließend ein bestimmtes Verhalten (Reaktion) auszulösen.

Vor allem sind solche Lehr- und Lernprozesse durch Frontalunterricht, Folienvorträge oder Tafelbilder, aber auch durch wiederholende Übungen der gleichen Art geprägt. Die behavioristische Lerntheorie gilt allerdings auch als theoretische Basis zahlreicher digitaler Lernanwendungen der früheren Generationen, wobei Lernziele in kleine Abschnitte unterteilt sind und Lernende durch einen fortlaufenden Wechsel zwischen Wissensvermittlung sowie dem Überprüfen des erworbenen Wissens in kleinen Aufgaben zu einem schnellen, oftmals jedoch kurzfristigen, Lernerfolg kommen (Kuhlmann und Sauter 2008). Die Aufgaben digitaler Medien beschränken sich hierbei insbesondere auf die Darstellung und Organisation von Wissen für das Lernen, also der Wissens(re)präsentation.

Der Hauptgrund dafür, dass die behavioristische Lerntheorie heutzutage häufig in Kritik steht, liegt darin, dass Lernprozesse in ihrer Komplexität durch ein Reiz- 
Reaktions-Modell nicht erfasst werden können und Menschen beim Lernen nicht nur passiv Wissen aufnehmen und Anweisungen befolgen. Der behavioristische Ansatz vernachlässigt demnach sowohl Motivation als auch Emotionen einzelner Lernender, aber auch, wie neues Wissen entsteht.

\subsubsection{Kognitivismus}

Die kognitivistische Lerntheorie (z. B. vertreten durch Köhler, Bandura und Bruner) betont im Gegensatz zum Behaviorismus nicht das Ergebnis, sondern den Lernprozess, der durch aktives Wahrnehmen, Erfahren und Erleben gekennzeichnet ist. Im Kern geht es also nicht mehr darum, auf bestimmte Stimuli zu reagieren. Stattdessen steht das Problemlösen und dafür benötigte Erlernen neuer Methoden und Verfahren im Vordergrund, so dass eine oder sogar mehrere korrekte Antworten generiert werden können. Neues Wissen wird dabei auf Basis bestehender Wissensstrukturen gebildet. Lernende verarbeiten aktiv Informationen und (re)organisieren diese, um eine mögliche Lösung für eine Problemstellung zu finden. Lehrende agieren beim Kognitivismus als Tutor/-innen und arbeiten didaktisch die notwendigen Informationen zur Problemlösung auf (Kuhlmann und Sauter 2008).

Digitale Medien unterstützen im Sinne der kognitivistischen Lerntheorie die Wissensvermittlung im Lernprozess durch Steuerung und Regelung. Bei diesem Ansatz finden die Lernprozesse meistens in laborähnlichen Situationen statt, wobei die zu lösenden künstlichen Problemstellungen auf das bereits vorhandene Wissen der Lernenden aufbauen. In Blended-Learning-Settings als Kombination des digitales Lernens mit klassischer Präsenzlehre, kann beispielsweise über eine digitale Lernanwendung eine problemorientierte Fallstudie bereitgestellt werden, die anschließend in der Gemeinschaft oder einem Workshop besprochen wird (Kuhlmann und Sauter 2008).

Auch die kognitivistische Lerntheorie steht in Kritik, häufig wird sie als zu einseitig angesehen. Angenommen wird, dass die Problemstellung objektiv gegeben ist sowie repräsentiert werden kann und nur auf eine Lösung wartet. Allerdings ist das in der Form häufig nicht der Fall. Zunächst müssen Probleme erkannt und formuliert werden, damit sie gelöst werden können. Insbesondere der Prozess der Problemgenerierung wird demnach vernachlässigt (Baumgartner und Payr 1997).

\subsubsection{Konstruktivismus}

Dies versucht die konstruktivistische Lerntheorie (z.B. vertreten durch Salomon, Bandura und Piaget) zu umgehen. Im Gegensatz zum Kognitivismus geht es beim Konstruktivismus nicht nur um das Lösen bereits existierender Herausforderungen, sondern auch um das Generieren neuer Probleme. Diese müssen in einem heutigen VUCA $^{1}$-Umfeld, geprägt durch häufige Veränderungen und sprunghafte Entwicklungen sowie unklaren Situationen, in denen vielfältige Elemente ineinander spielen, jedoch erst gefunden werden. Gerade darin und in dem kontextualisiertem und in

\footnotetext{
${ }^{1}$ VUCA ist ein Akronym für volatility (Unbeständigkeit), uncertainty (Unsicherheit), complexity (Komplexität) und ambiquity (Mehrdeutigkeit) zur Beschreibung veränderter Rahmenbedingungen.
} 
Situationen anwendbaren Wissen besteht nach der konstruktivistischen Theorie die Leistung. Lernende erschaffen bzw. konstruieren sich eine individuelle Repräsentation der Welt (Baumgartner und Payr 1997). Dabei ist Wissen nicht objektiv, sondern personengebunden und zeigt die selbst erlebte Wirklichkeit, d.h. persönliche Erfahrungen stehen im Vordergrund.

Lernen erfolgt demnach mit Inhalten, eingebettet in Situationen, die zu einer Wissenskonstruktion anleiten, wodurch eine Verbindung zwischen Lernenden und Lehrenden entsteht. Lehrende übernehmen in solch einem eigenverantwortlichen und sozialen Lernprozess die Rolle eines Coaches (Kuhlmann und Sauter 2008).

In der konstruktivistischen Lerntheorie kommt ganz besonders die Funktion der digitalen Medien als Wissenswerkzeug hinzu, welches die Kommunikation und Konstruktion des Wissens durch den Lernenden unterstützt. Demnach soll die IT das Lernen in Situationen ermöglichen. Moderne Kooperationsplattformen sind für diese Art des Lernens sinnvoll, da eine aktive Teilnahme der Lernenden an Kommunikationsund Konstruktionsprozessen gefördert wird (Kuhlmann und Sauter 2008).

\subsubsection{Konnektivismus}

Konnektivismus bildet keine eigenständige Lerntheorie, vielmehr kann es als eine Erweiterung der drei weit verbreiteten und bereits oben beschriebenen Lerntheorien angesehen werden, welche die gesellschaftlichen Veränderungen aufgrund der technologischen Entwicklungen im Lernen konsequent aufgreift und integriert. Konnektivismus beschreibt ein Lernen im Netzwerk. Zum Lernen ist demnach ein Herstellen von Verbindungen weitaus besser als zu versuchen, einzelne Konzepte zu verstehen (Siemens 2006).

Die Lerntheorien des Behaviorismus, Kognitivismus und Konstruktivismus werden den Veränderungen in der Gesellschaft und Wirtschaft laut Siemens (2006) nicht mehr gerecht. In der heutigen miteinander verbundenen und vernetzten Informations- und Wissensgesellschaft ist es weder ausreichend, noch möglich allein durch Begründungen und eigene Erfahrungen zu lernen. Insbesondere ist dies durch das exponentielle Wachstum des Wissens zu begründen, wodurch sich auch die Art zu lernen und kommunizieren grundlegend verändert hat. Lernen erfolgt kontextgebunden und aus einem Zusammenspiel zwischen Individuum und Umgebung. Demnach ist Lernen ein Prozess, der nicht nur von der eigenen Person, sondern auch maßgeblich vom Umfeld abhängig ist. Nur über ein Aufbauen von bedarfsgerechten Netzwerken ist es möglich das eigene Wissen immer aktuell und den Herausforderungen entsprechend zu sichern. Um das eigene Wissen aktuell zu halten, benötigen Lernende eine offene Lernumgebung, in der Interaktionen ermöglicht werden. Die Lehrenden übernehmen dabei die Rolle eines Mentors, wobei aktives Zuhören, Beobachten, Feedback geben und Beraten dazugehören (Kuhlmann und Sauter 2008).

Digitale Medien übernehmen im Konnektivismus die Funktion der Wissensvernetzung, wobei das Verknüpfen von Personen und Wissen in einem Netzwerk im Mittelpunkt steht. Durch einen ermöglichten Wissensaustausch und einer Kompetenzentwicklung in Netzwerken und über das Netz können digitale Medien so ein Lernen im Sinne des Konnektivismus optimal unterstützen. 


\subsection{Digitale Medien beeinflussen die Vielfalt an angebotenen Lernformaten}

Die digitale Transformation verändert nicht nur die Anforderungen des Lernens und auf welche Art und Weise gelernt wird, so wie aus den zuvor beschriebenen Lerntheorien und Aufgaben digitaler Medien hervorgeht, sondern auch die Vielfalt an möglichen Lernformaten und die damit einhergehenden Chancen. Digitale Medien haben sich in der Lehre von einem Werkzeug zum strategisch relevanten Werttreiber gewandelt. Sie fördern neben einem modernen, technologiegeleiteten Wissenserwerb auch den Bildungserfolg für Lehrende und Lernende. Des Weiteren verändern digitale Medien potenziell die Beziehung zwischen der Bildungsinstitution und ihren Lernenden, da diese als Schnittstelle fungieren und somit individuelle Werte schaffen können. Digitale Medien bieten die Chance zur persönlichen Bildungsbegleitung, ermöglichen durchgängige Bildungsketten sowie einen nachhaltigen Wissenserwerb. Außerdem können sie soziale sowie berufliche Vorteile mit sich bringen.

Durch eine starke Veränderung sowie Weiterentwicklung der technischen Möglichkeiten, entstehen neue Lernformate fast schon explosionsartig. Während vor einigen Jahren noch überwiegend mit Flipcharts, Pinnwänden, Overheadprojektoren oder reinen Powerpoint-Präsentationen Wissen vermittelt wurde, stehen Lehrenden heutzutage deutlich mehr Optionen bei der Gestaltung der Lehre zur Verfügung. Beispielsweise kann statt der klassischen Pinnwand zum Ideen sammeln innerhalb einer Lernendengruppe jetzt auch ein digitales Tool, wie z.B. Miro oder MURAL, verwendet werden, welches verteilten Teams ein effektives Zusammenarbeiten vom Brainstorming bis hin zur Planung und Ausführung von Arbeitsabläufen ermöglicht und durch nützliche Vorlagen noch zusätzlich unterstützt. Klassische und neuere

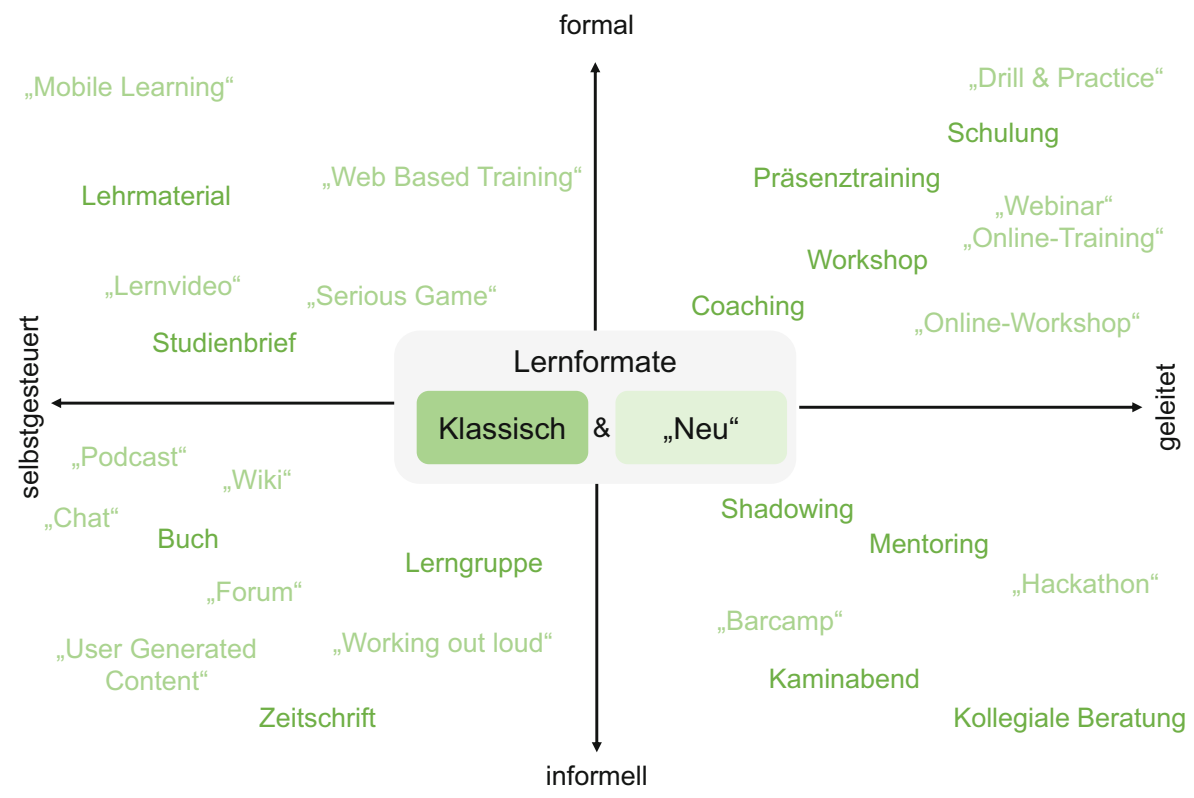

Abb. 2 Klassische und neue Lernformate (in Anlehnung an Sammet und Wolf (2019)) 
Möglichkeiten sowie damit begünstigte Lernformate können miteinander kombiniert werden und so dabei unterstützen das Lehren und Lernen besser zu machen, wobei z.B. mehr Spaß beim Lernen, eine erhöhte Motivation und ein gesteigerter Lernerfolg gemeint sind.

Abb. 2 gibt einen Überblick über verschiedene mögliche klassische und neuere Lernformate, wobei diese anhand der Lernprinzipien (formal oder informell sowie geleitet oder selbstgesteuert) unterteilt sind, so dass eine strukturierte Übersicht erst möglich ist. Während formales Lernen von einem Lehrenden heraus angeleitet und organisiert in Institutionen verläuft, geht informelles Lernen vom Lernenden aus und wird nicht von einem vorgegebenen Lehrplan bestimmt. Demnach erfolgt die Unterscheidung zwischen formalen und informellen Lernen anhand der Verantwortung sowohl für Lernziele, als auch -inhalte. Die Organisation und Aktivität des Lernens steht bei der Unterscheidung zwischen dem selbstgesteuertem und geleitetem Lernen im Fokus. Geleitet bedeutet, dass das Lernen von einem Lehrenden vorgegeben wird, wohingegen beim selbstgesteuertem Lernen eigenverantwortlich entschieden werden kann, wann, wo und wie gelernt wird (Sammet und Wolf 2019). Die im vorherigen Kapitel beschriebenen Lerntheorien Behaviorismus und Kognitivismus sind eher den geleiteten Lernprozessen zuzuordnen, während der Konstruktivismus und ergänzende Konnektivismus hauptsächlich selbstgesteuerte Lernprozesse abbilden.

Obwohl innerhalb der Abb. 2 die verschiedenen klassischen und neueren Lernformate in vier Quadranten unterteilt sind, ist die konkrete Ausgestaltung des Lehrens und Lernens durchaus differenzierter. Die Lernformate können innerhalb des Lernprozesses nämlich fließend ineinander übergehen. Beispielsweise ist die klassische Präsenzlehre formal und geleitet, d.h. die Lerninhalte und -ziele sind durch einen Lehrenden vorgegeben, genauso wie der Ablauf und die Organisation. Nun ist es jedoch so, dass gut und vor allem lernzielorientiert gestaltete Präsenzlehre immer auch informelle und selbstgesteuerte Phasen umfasst, wodurch verschiedene Lernformate geschickt miteinander vereint werden müssen, um den Lernprozess optimal zu fördern (Sammet und Wolf 2019).

Die nachfolgende Tab. 1 gibt einen Überblick darüber, was sich hinter den verschiedenen klassischen und neueren Lernformaten verbirgt, um ein Gefühl dafür zu bekommen, welche Lernformate möglich sind. Die aufgeführten Lernformate in Abb. 2, als auch Tab. 1 sind zwar gängige Möglichkeiten, um die Lehre zu gestalten, haben aber nicht den Anspruch auf Vollständigkeit. Diese orientieren sich an Sammet und Wolf (2019). Weitere Lernformate, die hier nicht vorgestellt und einsortiert werden sind dennoch denkbar und möglich, wie z.B. Lernen in einer Augmented oder Virtual Reality und Gamification.

Verschiedene in der Tab. 1 präsentierten Lernformate können miteinander kombiniert werden, um so unterschiedliche Interaktionen sowohl zwischen den Lernenden untereinander als auch zwischen den Lernenden und Lehrenden zu fördern. Um heutzutage erfolgreich zu lernen, womit beispielsweise das Erreichen eines hohen Lernerfolgs gemeint ist, ist die Integration von Interaktionen sowie Elementen der Zusammenarbeit sinnvoll und das nicht nur in Präsenz, sondern insbesondere auch im Digitalen.

Eine Kombination unterschiedlicher Lernformate ermöglicht es außerdem Nachteile des einen Lernformats mit den Vorteilen eines anderen Lernformats auszuglei- 
Tab. 1 Definitionen der klassischen und neueren Lernformate

\begin{tabular}{|c|c|c|c|}
\hline \multicolumn{2}{|c|}{ Klassische Lernformate } & \multicolumn{2}{|c|}{ Neuere digitale Lernformate } \\
\hline \multicolumn{4}{|c|}{ Formale und geleitete Lernprozesse } \\
\hline $\begin{array}{l}\text { Präsenz- } \\
\text { training }\end{array}$ & $\begin{array}{l}\text { Lehrende unterrichten alle Ler- } \\
\text { nenden zur gleichen Zeit an einem } \\
\text { bestimmten Ort }\end{array}$ & $\begin{array}{l}\text { Online- } \\
\text { Training }\end{array}$ & $\begin{array}{l}\text { Digitale Schulung, also Kurse } \\
\text { zur Wissensvermittlung }\end{array}$ \\
\hline Schulung & $\begin{array}{l}\text { Wissensvermittlung und Erfah- } \\
\text { rungsaustausch zu bestimmten } \\
\text { Themen }\end{array}$ & Webinar & $\begin{array}{l}\text { Online-Vortrag zu einer festen } \\
\text { Zeit mit interaktiven Elementen }\end{array}$ \\
\hline Workshop & $\begin{array}{l}\text { Kleine Gruppe entwickelt z.B. neue } \\
\text { Strategien oder Problemlösungen }\end{array}$ & $\begin{array}{l}\text { Online- } \\
\text { Workshop }\end{array}$ & $\begin{array}{l}\text { Kleine Gruppe entwickelt di- } \\
\text { gital z. B. neue Strategien oder } \\
\text { Problemlösungen }\end{array}$ \\
\hline Coaching & $\begin{array}{l}\text { Professionelle Beratung, Begleitung } \\
\text { und Unterstützung }\end{array}$ & $\begin{array}{l}\text { Drill \& } \\
\text { Practice }\end{array}$ & $\begin{array}{l}\text { Lern-Software charakterisiert } \\
\text { durch eine systematische Wie- } \\
\text { derholung von Übungen }\end{array}$ \\
\hline \multicolumn{4}{|c|}{ Informelle und geleitete Lernprozesse } \\
\hline $\begin{array}{l}\text { Kollegiale } \\
\text { Beratung }\end{array}$ & $\begin{array}{l}\text { Beratungsgespräch in einer Gruppe } \\
\text { gleichberechtigter Partner, wobei } \\
\text { jeder eine festgelegte Rolle hat }\end{array}$ & Hackathon & $\begin{array}{l}\text { Kollaborativ in kurzer Zeit } \\
\text { eine kreative Software- oder } \\
\text { Hardwarelösung entwickeln }\end{array}$ \\
\hline Mentoring & $\begin{array}{l}\text { Erfahrene Person (Mentor) gibt } \\
\text { Wissen an unerfahrene Person } \\
\text { weiter }\end{array}$ & Barcamp & $\begin{array}{l}\text { Offene Konferenz, bei der Teil- } \\
\text { nehmende zu Beginn selbst } \\
\text { Programmpunkte bestimmen }\end{array}$ \\
\hline Shadowing & $\begin{array}{l}\text { Person begleitet eine erfahrene } \\
\text { Person eine gewisse Zeit bei seinen } \\
\text { Tätigkeiten (quasi als Schatten) }\end{array}$ & - & - \\
\hline Kaminabend & $\begin{array}{l}\text { Informelles Treffen, um Austausch } \\
\text { von Ideen, Wissen etc. zu fördern }\end{array}$ & - & - \\
\hline \multicolumn{4}{|c|}{ Informelle und selbstgesteuerte Lernprozesse } \\
\hline Lerngruppe & $\begin{array}{l}\text { Zusammenschluss von Lernenden, } \\
\text { um gemeinsam zu lernen }\end{array}$ & Chat & $\begin{array}{l}\text { Digitale Kommunikation zwi- } \\
\text { schen zwei oder mehreren Per- } \\
\text { sonen }\end{array}$ \\
\hline Zeitschrift & $\begin{array}{l}\text { Regelmäßig erscheinendes Magazin } \\
\text { zu einem bestimmten Thema }\end{array}$ & Podcast & $\begin{array}{l}\text { Digitale Audio- und Videoinhal- } \\
\text { te als Gespräche }\end{array}$ \\
\hline Buch & $\begin{array}{l}\text { Veröffentlichter literarischer oder } \\
\text { wissenschaftlicher Text }\end{array}$ & Forum & $\begin{array}{l}\text { Digitaler Austausch zum Lö- } \\
\text { sen von Problemen in einem } \\
\text { geeigneten Personenkreis }\end{array}$ \\
\hline- & - & Wiki & $\begin{array}{l}\text { Selbstbearbeitete digitale Infor- } \\
\text { mationen und Beiträge zu einem } \\
\text { bestimmten Thema }\end{array}$ \\
\hline- & - & $\begin{array}{l}\text { User Ge- } \\
\text { nerated } \\
\text { Content }\end{array}$ & $\begin{array}{l}\text { Lernende erstellen digitale } \\
\text { Lerninhalte }\end{array}$ \\
\hline- & - & $\begin{array}{l}\text { Working } \\
\text { out loud }\end{array}$ & $\begin{array}{l}\text { Wissen z. B. über soziale Me- } \\
\text { dien oder Wikis teilen und so } \\
\text { sichtbar machen }\end{array}$ \\
\hline
\end{tabular}


Tab. 1 (Fortsetzung)

\begin{tabular}{llll}
\hline \multicolumn{2}{l}{ Klassische Lernformate } & Neuere digitale Lernformate \\
\hline $\begin{array}{l}\text { Formale und selbstgesteuerte Lernprozesse } \\
\text { Studienbrief } \\
\text { Lerninhalte zu bestimmten Thema } \\
\text { mit Übungsaufgaben }\end{array}$ & $\begin{array}{l}\text { Mobile } \\
\text { Learning } \\
\text { Web } \\
\text { Based } \\
\text { Training }\end{array}$ & $\begin{array}{l}\text { Lernen mit mobilen Endgeräten } \\
\text { (z. B. Smartphone oder Tablet) } \\
\text { Digitales Lernprogramm zum } \\
\text { (a)synchronen Lernen }\end{array}$ \\
& $\begin{array}{l}\text { Lernvideo } \\
\text { talveranstaltung }\end{array}$ & $\begin{array}{l}\text { Videos, die Lerninhalte vermit- } \\
\text { teln }\end{array}$ \\
- & - & $\begin{array}{l}\text { Serious } \\
\text { Game vollständiges Spiel mit } \\
\text { festen Regeln und Zielen zum } \\
\text { Lernen }\end{array}$ \\
\hline
\end{tabular}

chen (Sammet und Wolf 2019). Jeder Mensch lernt anders optimal und durch eine raffinierte Kombination der Lernformate können viele Lernende (mit verschiedenen bevorzugten Lernstrategien und unterschiedlich ausgeprägten Persönlichkeitstypen) in ihren individuellen Lernprozessen profitieren.

Für eine Unterstützung guter Lehre müssen Lernformate die Lernenden dazu bringen verschiedene fachliche, forschungsorientierte, gesellschaftliche und persönliche Kompetenzen auf unterschiedlichen Ebenen zu erwerben. Dazu sind digitale Lernformate immer dann sinnvoll, wenn sie gegenüber den physischen, also eher klassischen, Lernformen Vorteile aufweisen. Flipped Classroom ermöglicht beispielsweise digital den eigenständigen Wissenserwerb sowie physisch die Anwendung und Reflektion des Wissens. Mit Gamification kann beispielsweise eine eigenständige $\mathrm{Zu}$ sammenarbeit in Gruppen sowie die Motivation zu lernen gestärkt werden (Eckardt et al. 2015). Im Mobile Learning gibt es z. B. Konzepte, bei denen Lernende sich untereinander kennenlernen und miteinander verbinden, wobei ein unterstütztes PeerLearning zu einer Verbesserung der Lernumgebung und der sozialen Kontakte führt (Kallookaran und Robra-Bissantz 2019). Ambient Learning ermöglicht z.B. eine Interaktion mit der realen Welt über digitale Schnittstellen, wodurch weiterführende Informationen zu einem Zeitpunkt, an einem bestimmten Ort, in einem angemessenen Tempo und Kontext, der für den einzelnen Lernenden am besten geeignet, bereitgestellt werden (Robra-Bissantz 2006). Hinsichtlich des Erwerbs unterschiedlicher Kenntnisse und Kompetenzen sind verschiedene Lernformate sinnvoll, wobei es immer auf die anvisierten Lernziele ankommt. Wenn es z. B. darum geht Fakten zu lernen, sind behavioristische Ansätze geeignet. Beispielsweise gibt es dabei die Möglichkeit in einem virtuellen Gedächtnispalast mittels eines Head-MountedDisplays zu lernen (Huttner und Robra-Bissantz 2017). Für andere Lernziele eignen sich wiederum andere Ansätze.

Die Wissens- und Kompetenzvermittlung an Hochschulen, Schulen sowie anderen (betrieblichen) Aus- und Weiterbildungseinrichtungen befindet sich bereits seit längerem im Wandel, wobei Corona diesen noch zusätzlich beschleunigt hat. Während Lehrende an einigen Einrichtungen schon immer hauptsächlich neuere Lernformate einsetzen, lassen Lehrende an anderen Einrichtungen weitestgehend in Präsenz mit eher klassischen Formaten lernen. Nachfolgend widmet sich dieser Beitrag vor die- 
sem Hintergrund deshalb der Thematik, wie Corona das Lernen an Hochschulen, Schulen sowie in der Aus- und Weiterbildung verändert hat.

\section{Digitales Lernen und Lehren an Hochschulen}

Die Digitalisierung des Lernens und Lehrens war bereits vor Corona an Hochschulen ein aktuelles Thema. Einige, vor allem virtuelle, Hochschulen bieten schon immer reine Online-Lehre an, insbesondere aus Gründen der Zeit- und Ortsunabhängigkeit. Dabei nutzen Lehrende beispielsweise das eher klassische Lernformat der Studienbriefe, um ihren Studierenden neues Wissen zu vermitteln. Eine Interaktion und Kommunikation mit Studierenden, beides zentrale Bestandteile eines gelungenen Lehr- und Lernprozesses (Mayrberger 2017), findet dabei jedoch nur selten statt. Andere Hochschulen wiederum setzen hauptsächlich auf Präsenzlehre, sowohl in angebotenen Vorlesungen als auch begleitenden Seminaren und Praktika. Lediglich punktuell werden in Lehrveranstaltungen an Hochschulen geschickt klassische und neue Lernformate miteinander kombiniert, um Studierende beim Lernen zu unterstützen und nicht mehr nur den Fokus auf eine erhöhte Digitalisierung der Lehre zu legen, sondern auch im Virtuellen Werte für die Lernenden zu schaffen, die sich beispielweise in ermöglichten Interaktionen zeigen, die zu mehr Engagement und Partizipation anregen.

Mit Beginn von Corona standen Hochschulen plötzlich vor enormen Herausforderungen. Einzelne digitale Lernangebote reichten nicht mehr aus. Stattdessen musste innerhalb weniger Wochen das komplette Lehrangebot umgestellt werden. Dies bedeutete für Studierende und Lehrende gleichermaßen nahezu vollständig auf Präsenzunterricht zu verzichten und die gesamte Lehre, einschließlich aller Vorlesungen, begleitender Übungen, Seminare, Praktika aber auch Prüfungen, virtuell zu organisieren.

In einer Studie mit mehr als 11.000 teilgenommenen Studierenden sowie 1800 Lehrenden, durchgeführt vom Stifterverwand für die Deutsche Wissenschaft e. V. in Kooperation mit McKinsey, wurde untersucht, wie die Hochschulen mit der besonderen Situation umgegangen sind (Winde et al. 2020). Die abrupte Umstellung auf eine nahezu vollständige digitale Lehre hat dazu geführt, dass im Sommersemester 2020 bereits $91 \%$ der durchgeführten Lehrveranstaltungen digital abliefen, wohingegen im vorherigen Wintersemester 2019/20 gerade einmal $12 \%$ ein digitales Lernformat wählten. Größtenteils wird die Umstellung der Lehre ins Digitale positiv bewertet, sowohl auf Seiten der Lehrenden als auch auf Seiten der Studierenden. Allerdings gibt es zwischen den verschiedenen Veranstaltungsformen Unterschiede. Während digitale Vorlesungen von über $80 \%$ eher positiv wahrgenommen werden, zeigt sich insbesondere bei Laborarbeiten ein gegensätzliches Bild. Bei Seminaren und Übungen, wie sie neben Vorlesungen häufig vertreten sind, bewerten Lehrende und Studierende diese als digitales Format zu etwa 50\% positiv (Winde et al. 2020). Hierzu ist allerdings anzumerken, dass die befragten Studierenden und Lehrenden aus allen Fachgebieten (z. B. Humanmedizin oder Kunst und Sport) kommen und eine Auswertung insgesamt, nicht aber bezüglich einzelner Fachbereiche erfolgt 
ist. Es ist demnach gut möglich, dass digitale Lehrveranstaltungen von einzelnen Fachgebieten leicht verändert wahrgenommen werden.

In einer weiteren Online-Befragung, an der sich fast 28.600 Studierende beteiligt haben und die in Zusammenarbeit zwischen dem Deutschen Zentrum für Hochschulund Wissenschaftsforschung (DZHW) sowie der AG Hochschulforschung der Universität Konstanz durchgeführt wurde, wurde u. a. der Einsatz digitaler Lehr- und Lernformate untersucht, aber auch die Auswirkungen von Corona auf die Studiensituation (Marczuk et al. 2021). Die Mehrheit der befragten Studierenden (>75\%) gibt an, dass mindestens die Hälfte ihrer belegten Lehrveranstaltungen als Videokonferenz (z. B. in Form eines Webinars) stattgefunden haben. Außerdem haben etwas mehr als die Hälfte der Studierenden Lehrmaterialien bereitgestellt bekommen und $54 \%$ der Umfrage-Teilnehmenden haben darüber hinaus zu lösende Aufgaben erhalten. Audioaufzeichnungen (z. B. Podcasts) wurden hingegen äußerst selten zum Lernen bereitgestellt. Ebenfalls geht aus der Studie hervor, dass die Lehrveranstaltungen nicht nur einzelne Lernformate verwendet haben. Vielmehr wurde versucht verschiedene Formate in einer Lehrveranstaltung sinnvoll zu integrieren. So wurden beispielsweise häufig Lehrmaterialien und begleitende Aufgaben ergänzend zur Videokonferenz online herausgegeben (Marczuk et al. 2021). Über weitere genutzte Lernformate, sowohl klassisch als auch neuere, wie sie in Tab. 1 vorgestellt sind, können aus der durchgeführten Umfrage keine Erkenntnisse gewonnen werden. Es ist jedoch anzunehmen, dass die in der Studie erhobenen Lehr- und Lernformate die am häufigsten genutzten in der Corona-Pandemie sind und weitere eher selten zum Einsatz gekommen sind.

Bei der Betrachtung der Konsequenzen für die Studiensituation haben sich Marczuk et al. (2021) auf die Kontaktmöglichkeiten und die individuelle Lernsituation beschränkt. Insgesamt zeigen die Ergebnisse, dass sich diese im Allgemeinen während Corona eher verschlechtert haben. Insbesondere die Kontaktsituation ist schwieriger geworden. Dies zeigt eine wahrgenommene Verschlechterung seitens der Studierenden beim Austausch in Lerngruppen (77\%), bei der Kommunikation mit Lehrenden (61\%) aber auch bei den Kontakten zu anderen Studierenden (86\%). Eine gute soziale Integration ist jedoch wesentlich für den Erfolg im Studium (Schaeper 2020), genauso wie eine hohe Interaktion und Kommunikation wesentliche Elemente für einen erfolgreichen Lernprozess sind (Mayrberger 2017), ebenso wie Möglichkeiten der Kooperation/Kollaboration (Eckardt \& Finster 2019).

Damit durch die veränderten Studienbedingungen mittelfristig nicht die Studienleistungen und die Zufriedenheit der Studierenden sinken, was mit höheren Studienabbruchrisiken verbunden ist (Marczuk et al. 2021), sind schnellstmöglich noch mehr Möglichkeiten für Interaktionen zwischen den Studierenden und mit den Lehrenden, aber auch Möglichkeiten der Zusammenarbeit in Lehrkonzepte einzubinden. Einige denkbare Lernformate sind dafür in Tab. 1 aufgeführt (z. B. Chat, Foren, Hackathons). Es ist aktuell bereits so, dass an zahlreichen Hochschulen schon vereinzelt Lehrkonzepte eingesetzt werden, die Interaktionen und Formen der Zusammenarbeit gezielt digital fördern (z.B. AC:DC-Agile and Collaborative Digital Classroom von Grogorick und Lamprecht (2021)), dennoch besteht diesbezüglich in Hinblick auf eine Betrachtung des Lernens und Lehrens im Allgemeinen an den Hochschulen noch Verbesserungspotenzial. 
Lediglich $52 \%$ der befragten Lehrenden aus der Studie von Winde et al. (2020) haben angegeben, dass die eigene Hochschule seit Corona über digitale Lehrkonzepte verfügt. Das sind zwar 36\% (an Hochschulen) bzw. 27\% (an Fachhochschulen) mehr als vor Ausbruch von Corona, aber dennoch nicht genug in Anbetracht dessen, dass die Lehre in Präsenz zeitweise nahezu vollständig zum Erliegen kam. Die Digitalisierung des Lernens und Lehrens hat während Corona dennoch Fortschritte gemacht und insbesondere gezeigt, dass digitale Lernformate dauerhaft, wenn auch als Ergänzung zur Präsenzlehre möglich sind. Gemachte Erfahrungen müssen jedoch erst noch systematisch gesammelt und ausgewertet werden, um bisherige Lehrkonzepte anzupassen und weiterzuentwickeln, so dass eine ganzheitliche Vision zur Förderung des (digitalen) Lernens und Lehrens and Hochschulen nach und nach gestaltet sowie umgesetzt werden kann, die Lern- und Studienerfolg fördert.

\section{Digitales Lernen und Lehren an Schulen}

Vor Corona war das Lernen und Lehren an Schulen ähnlich zu den Lehrveranstaltungen an Hochschulen weitestgehend durch Präsenzveranstaltungen geprägt, wenn auch in kleineren Gruppen von 15-30 Schülerinnen und Schülern. Die Präsenzlehre wurde punktuell dann noch durch den Einsatz digitaler Medien ergänzt, beispielsweise wurde gelegentlich ein Video zur besseren Veranschaulichung gezeigt. Allerdings erfolgte dies relativ selten und auch nur ergänzend zum klassischen Face-to-FaceUnterricht.

Die mit Corona einhergehenden Schulschließungen haben die Digitalisierungsdefizite der deutschen Bildungslandschaft schonungslos offenbart. Nicht nur die digitale Infrastruktur, inklusive mangelnder technischer Ausstattung und funktionstüchtiger Geräte, ist im Schulwesen unzureichend. Auch fehlen Lehrkonzepte für den digitalen Unterricht sowie Lehrende, die im Umgang des digitalen Lernens und Lehrens geschult sind.

Eine Umfrage der Deutschen Telekom Stiftung unter mehr als 1000 Schülerinnen und Schülern der Klassenstufen fünf bis zehn hat ergeben, dass 52\% der Meinung sind durch die coronabedingten Schulschließungen mit dem Lernstoff etwas im Rückstand zu sein. $27 \%$ der Teilnehmenden gab sogar an, deutlich mit dem Lernstoff im Rückstand zu sein. Das zeigt, dass das Lernen und Lehren durch Corona deutlich beeinträchtigt wurde. Lediglich $10 \%$ haben angegeben keinen Lernrückstand zu empfinden, die restlichen $11 \%$ sind diesbezüglich unentschieden (Deutsche Telekom Stiftung 2021).

Die Art und Weise, wie Lehr- und Lernmaterialien während und nach dem ersten Lockdown 2020 bereitgestellt wurden, variiert zwischen den verschiedenen Schultypen (Grundschule, Gymnasium und Haupt-/Real-/Gesamtschule), wie eine Studie ergab, für die 1812 Haushalte mit schulpflichtigen Kindern befragt wurden (Huebener et al. 2020). In der Grundschule erhielten die Lernenden ihre Materialien zu $17 \%$ im ersten Lockdown per Videokonferenz, und danach waren es $24 \%$. Am Gymnasium haben bereits im ersten Lockdown $36 \%$ per Videokonferenz gelernt, wobei dieser Prozentsatz in der Zeit danach auf 57\% angestiegen ist. An Haupt-, Real- und Gesamtschulen ist der Prozentsatz mit 25 bzw. $23 \%$ sowohl während des 
ersten Lockdowns als auch danach nahezu identisch geblieben. Im Vergleich zu den Hochschulen, an denen im Sommersemester 2020 (zum Zeitpunkt des ersten Lockdowns) bereits über $90 \%$ der Lehrveranstaltungen digital abgehalten wurden, wird bei den verschiedenen Schultypen deutlich weniger mittels Videokonferenz gelernt. Stattdessen haben die Schülerinnen und Schüler ihre Lernmaterialien häufig zum Teil vor der Schulschließung erhalten oder per E-Mail oder Cloud, teilweise jedoch sogar per Post.

Mediale Lernangebote, welche die Schülerinnen und Schüler neben den bereitgestellten Lernmaterialien durch die Lehrenden während dieser Zeit verwendet haben, sind YouTube (über $80 \%$ ), Wikipedia (58\%) und Dokumentationen sowie Wissenssendungen im Fernsehen, Schulsendungen im Fernsehen oder Internet und Onlinebibliotheken der Büchereien mit jeweils weniger als $30 \%$ (mpfs 2020).

Über $73 \%$ der 2038 Befragten aus einer weiteren Studie haben angegeben, dass durch Corona bereits ein Fortschritt im Bereich der Digitalisierung des Bildungswesens stattgefunden hat. Außerdem sind Dreiviertel der Teilnehmenden der Meinung, dass die Lehr- und Lernkonzepte in der Zukunft noch deutlich mehr auf die digitale Welt angepasst werden müssen, und diese dann in Schulen öfters einfach mal ausprobiert und ggf. auch wieder verworfen werden sollten. Knapp $80 \%$ wollen, dass Lehrende künftig Fortbildungen im Bereich digitaler Lernformate erhalten sollen, damit das Lehren und Lernen insgesamt digitaler wird (Initiative D21 2021).

\section{Digitales Lernen und Lehren in der Aus- und Weiterbildung}

In der Wirtschaft hat Corona erhebliche Auswirkungen gehabt und zu deutlichen Beeinträchtigungen geführt, beispielsweise durch unterbrochene Lieferketten sowohl beim privaten Konsum, aber auch im Export. Doch auch in der betrieblichen Ausund Weiterbildung, die insbesondere wertvoll ist, um in einer Welt, die sich ständig ändert, als Unternehmen wettbewerbsfähig zu bleiben, hat Corona einige Herausforderungen bedeutet.

An einer Studie des Instituts der deutschen Wirtschaft haben 377 Unternehmen teilgenommen und ihre Weiterbildung während Corona bewertet (Flake et al. 2020). Größtenteils wurden die Weiterbildungsaktivitäten der Unternehmen während dieser Zeit aufrechterhalten. Bei knapp der Hälfte der Befragten hat sich die Weiterbildung im Vergleich zu vorher nicht verändert. Bei einem Viertel ist das Angebot gesunken, wohingegen die Weiterbildung bei $12 \%$ sogar gestiegen ist. Tatsächlich ist es so, dass insbesondere große Unternehmen mit mehr als 100 Mitarbeitenden ihre Weiterbildungsaktivitäten etwas reduziert haben und kleinere sowie mittelständische Unternehmen (mit 20 bis 99 Mitarbeitenden) die unternehmensinternen Weiterbildungsangebote sogar ausgebaut haben. vermutlich um den bereits bestehenden Fachkräftemangel nicht noch weiter zu fördern und vorhandene Mitarbeitende zu halten (Flake et al. 2020).

Genauso wie an Hochschulen und Schulen konnten die Aus- und Weiterbildungsangebote während Corona aufgrund von Kontaktbeschränkungen weitestgehend nicht in Präsenz stattfinden. Folglich ist der Anteil an genutzten digitalen 
Lernangeboten in den Unternehmen stark angestiegen (um 36\%). Nur 4,8\% verzeichneten einen Rückgang (Flake et al. 2020).

Da viele Unternehmen bereits vor Beginn von Corona Weiterbildungsaktivitäten begonnen oder zumindest geplant haben, ist es interessant $\mathrm{zu}$ schauen, inwiefern diese fortgeführt werden konnten. Knapp ein Drittel der befragten Unternehmen gab an, dass die Weiterbildung (fast) vollständig ins Digitale überführt wurde. Ein weiteres Drittel konnte dafür jedoch kein adäquates Angebot identifizieren. Ca. 18\% wollen mit der Weiterbildung allerdings erst dann fortfahren, wenn diese wieder in Präsenz möglich ist und 15,9\% haben die Weiterbildungsaktivitäten aus sonstigen Gründen abgesagt (Flake et al. 2020).

Um die Weiterbildungsaktivitäten im Allgemeinen zu erhöhen, wünschen sich die Unternehmen mehr Unterstützung. Einerseits betrifft dies eine Ausweitung der finanziellen Förderung $(47,4 \%)$, andererseits besteht ein hoher Informationsbedarf zu möglichen (digitalen) Lernformaten (39,3\%). Damit Lernangebote überhaupt digital in Unternehmen zum Einsatz kommen ist ein gemeinsamer Austausch und vor allem das Erleben von Best-Practice-Beispielen ein wesentlicher Motivationsfaktor (Flake et al. 2020).

\section{Schlussbemerkungen}

Corona hat insgesamt zwar zu einer erhöhten Digitalisierung des Lernens und Lehrens sowohl an Hochschulen, Schulen, als auch anderen Aus- und Weiterbildungseinrichtungen geführt, gleichzeitig aber auch Defizite bisheriger Bemühungen offenbart.

Während allgemein festgehalten werden kann, dass die Hochschulen in Bezug auf digitales Lernen und Lehren zwar bereits etwas fortgeschrittener sind als Schulen sowie Aus- und Weiterbildungseinrichtungen, mangelt es nach bestem Wissen dennoch an allen betrachteten Bildungseinrichtungen weiterhin an (digitalen) Lehrkonzepten, die Interaktionen gezielt fördern und insbesondere ein gemeinsames, vernetztes Lernen im Sinne der sich weiterentwickelnden Lerntheorien und Möglichkeiten der verschiedenen Lernformate unterstützen.

Das digitale Lernen an den betrachteten Bildungsinstitutionen macht deutlich, dass die meisten Konzepte, die derzeit laufen keiner aktuellen Lerntheorie geschuldet sind und deshalb auch in Folge von Corona noch nicht vom digitalen Lernen als das neue Normal gesprochen werden kann. Jedenfalls ist noch viel mehr möglich, da digitales Lernen und Lehren mehr sind als traditionelle Lernprozesse unabhängig von Zeit und Raum. Vielmehr geht es darum den Lernerfolg geschickt durch innovative postdigitale Lehrszenarien in hybriden Lernräumen zu fördern, wobei die Vorteile aus dem digitalen Lernen mit den Vorteilen aus der analogen Lehre verbunden sein müssen.

Damit (digitales) Lernen und Lehren nicht nur durch Corona einen kurzen Aufschwung erfährt, sondern auch in Zukunft zu einer Ausweitung sowie Weiterentwicklung der Angebote an allen Bildungseinrichtungen führt, wobei klassische mit neueren Lernformaten in Kombination zur Förderung eines hohen Lernerfolgs eingesetzt werden, ist ein Austausch von Erfahrungswerten wesentlich. Getreu des Mottos 
„Wissen teilen ist Macht“", was auch dem konnektivistischen Ansatz entspricht, sollte zunächst ein bewusster Austausch, also eine Vernetzung zwischen den verschiedenen Bildungseinrichtungen erfolgen, so dass gemeinsam voneinander gelernt werden kann und für die unterschiedlichen Bedürfnisse (digitale) Lehr- und Lernkonzepte gestaltet werden können, die Lernende in ihren individuellen Lernprozessen beim Erreichen der Lernziele unterstützen.

Lernen und Lehren müssen vor diesem Hintergrund anpassungsfähig sein und flexibel gestaltet werden können, damit Lehrende nicht nur in unvorhersehbaren Situationen, wie z. B. in Folge von Corona, reagieren können und Lehrveranstaltungen anpassen, sondern auch um Lernende insgesamt besser beim Erreichen der Lernziele zu unterstützen. Die ermöglichten Lernformate, wobei sowohl die klassischen, als auch die neueren gemeint sind, und die Kombination dieser, sind dabei nur ein erster Schritt. Lernende lernen auf verschiedene Art und Weise, weshalb insbesondere personalisiertes Lernen, beispielsweise durch die Unterstützung von künstlicher Intelligenz, in den nächsten Jahren das Lernen und Lehren verändern wird. Einige Lernende erreichen z. B. durch Serious Games einen hohen Lernerfolg, während andere ggf. besser in einem Präsenz-Workshop lernen. Das Ziel guter Lehre sollte es daher sein flexibel zu sein und entsprechend der individuellen Anforderungen Lernangebote ad-hoc zu schaffen, welche die Lernenden individuell und personalisiert beim Erreichen hoher Lernerfolge unterstützen.

Funding Open Access funding enabled and organized by Projekt DEAL.

Open Access Dieser Artikel wird unter der Creative Commons Namensnennung 4.0 International Lizenz veröffentlicht, welche die Nutzung, Vervielfältigung, Bearbeitung, Verbreitung und Wiedergabe in jeglichem Medium und Format erlaubt, sofern Sie den/die ursprünglichen Autor(en) und die Quelle ordnungsgemäß nennen, einen Link zur Creative Commons Lizenz beifügen und angeben, ob Änderungen vorgenommen wurden.

Die in diesem Artikel enthaltenen Bilder und sonstiges Drittmaterial unterliegen ebenfalls der genannten Creative Commons Lizenz, sofern sich aus der Abbildungslegende nichts anderes ergibt. Sofern das betreffende Material nicht unter der genannten Creative Commons Lizenz steht und die betreffende Handlung nicht nach gesetzlichen Vorschriften erlaubt ist, ist für die oben aufgeführten Weiterverwendungen des Materials die Einwilligung des jeweiligen Rechteinhabers einzuholen.

Weitere Details zur Lizenz entnehmen Sie bitte der Lizenzinformation auf http://creativecommons.org/ licenses/by/4.0/deed.de.

\section{Literatur}

Baumgartner P, Payr S (1997) Erfinden lernen. In: Müller A, Müller KH, Stadler F (Hrsg) Konstruktivismus und Kognitionswissenschaft: Kulturelle Wurzeln und Ergebnisse. Springer, Berlin, Heidelberg, New York, S 89-106

Deutsche Telekom (2021) Lernen in Zeiten von Corona. Eine repräsentative Befragung von Schülern der Klassen 5 bis 10 und Eltern von Kindern dieser Altersgruppe. https://www.telekom-stiftung. de/sites/default/files/files/media/publications/Lernen-in-Zeiten-von-Corona-Zusammenfassung.pdf. Zugegriffen: 14. Sep. 2021

Eckardt L, Finster R (2019) Kollaboration oder Wettbewerb: Ein Vergleich der Motivation beim Gamebased Learning. HMD 56:83-93. https://doi.org/10.1365/s40702-018-00481-7

Eckardt L, Siemon D, Robra-Bissantz S (2015) GamEducation - Spielelemente in der Universitätslehre. HMD 52:915-925. https://doi.org/10.1365/s40702-015-0177-6 
Flake R, Seyda S, Werner D (2020) Weiterbildung während der Corona-Pandemie. Institut der deutschen Wirtschaft. https://www.kofa.de/service/bestellshop/detailseite/news/kofa-kompakt-weiterbildungwaehrend-der-corona-pandemie. Zugegriffen: 14. Sep. 2021

Grogorick L, Lamprecht J (2021) AC:DC - Agiles und kollaboratives digitales Klassenzimmer. HMD 58:858-869. https://doi.org/10.1365/s40702-021-00736-w

Huebener M, Spieß CK, Zinn S (2020) SchülerInnen in Corona-Zeiten: Teils deutliche Unterschiede im Zugang zu Lernmaterial nach Schultypen und -trägern. DIW Berlin. https://www.diw.de/documents/ publikationen/73/diw_01.c.804515.de/20-47-1.pdf. Zugegriffen: 14. Sep. 2021

Huttner JP, Robra-Bissantz S (2017) An immersive memory palace: supporting the method of loci with virtual reality. In: Proceedings of the 23rd Americas Conference on Information Systems (AMCIS)

Initiative D21 (2021) Digitaler Unterricht während Corona: Erfahrungen von Lehrkräften, SchülerInnen und Eltern. https://initiatived21.de/digitaler-unterricht-lehrkraefte-bleiben-zu-oft-auf-sich-gestellt/. Zugegriffen: 14. Sep. 2021

Kallookaran M, Robra-Bissantz S (2019) C4mpUs: mehr Interaktion durch mobile Begleitung. In: Kauffeld S, Othmer J (Hrsg) Handbuch Innovative Lehre. Springer, Wiesbaden https://doi.org/10.1007/ 978-3-658-22797-5_15

Kerres M (2013) Mediendidaktik - Konzeption und Entwicklung mediengestützter Lernangebote. Oldenburg, München https://doi.org/10.1524/9783486736038

Kuhlmann A, Sauter W (2008) Innovative Lernsysteme: Kompetenzentwicklung mit blended learning und social Software. Springer, Berlin, Heidelberg, New York https://doi.org/10.1007/978-3-540-77831-8

Marczuk A, Multrus F, Lörz M (2021) Die Studiensituation in der Corona-Pandemie: Auswirkungen der Digitalisierung auf die Lern- und Kontaktsituation von Studierenden. DZHW Brief, 1. https:// www.die-studierendenbefragung.de/fileadmin/user_upload/publikationen/dzhw_brief_01_2021.pdf. Zugegriffen: 14. Sep. 2021

Mayrberger K (2017) Student Engagement und Digitalisierung - Erfolgsfaktoren für eine zeitgemäße Lehre und Administration an der Hochschule. In: DZHW (Hrsg) Jahrestagung der Gesellschaft für Hochschulforschung: Digitalisierung der Hochschulen: Forschung, Lehre, Administration. Hannover, S 20-21 (https://www.dzhw.eu/gfhf2017/pdf/Abstractband_GfHf2017.pdf)

mpfs (Medienpädagogischer Forschungsverbund Südwest) (2020) Lernen und Freizeit in der Corona-Krise. https://www.mpfs.de/fileadmin/files/Studien/JIM/JIMplus_2020/JIMplus_2020_Corona.pdf. Zugegriffen: 14. Sep. 2021

Robra-Bissantz S (2006) Ambient Learning. In: Hohenstein A, Wilbers K (Hrsg) Handbuch E-Learning. Verlag deutscher Wirtschaftsdienst, Köln, S 1-20

Sammet J, Wolf J (2019) Die „Learning Revolution“. In: Sammet J, Wolf J (Hrsg) Vom Trainer zum agilen Lernbegleiter. Springer, Berlin, Heidelberg, New York, S 1-21 https://doi.org/10.1007/978-3-66258510-8_1

Schaeper H (2020) The first year in higher education: the role of individual factors and the learning environment for academic integration. High Educ 79(1):95-110. https://doi.org/10.1007/s10734-01900398-0

Siemens G (2006) Knowing knowledge. Lulu.com

Winde M, Werner SD, Gumbmann B, Hieronimus S (2020) Hochschulen, Corona und jetzt? Stifterverband. https://www.stifterverband.org/medien/hochschulen-corona-und-jetzt. Zugegriffen: 14. Sep. 2021 